\title{
Formas de ureia e doses de nitrogênio em cobertura na qualidade fisiológica de sementes de trigo ${ }^{1}$
}

\author{
André Mateus Prando ${ }^{2 *}$, Claudemir Zucareli², Vanoli Fronza ${ }^{3}$, \\ Eliege Aparecida de Paiva Oliveira ${ }^{2}$, Barbara Panoff ${ }^{4}$
}

\begin{abstract}
RESUMO - A adubação nitrogenada, além de aumentar a produtividade, pode favorecer a qualidade fisiológica de sementes. O objetivo do trabalho foi avaliar o efeito de doses de adubação nitrogenada em cobertura e de diferentes formas de ureia na qualidade fisiológica de sementes de genótipos de trigo. Foram avaliadas sementes de três genótipos (BRS 208, BRS Pardela e IWT 04008) cultivados sob quatro doses de nitrogênio em cobertura (0, 40, 80 e 120 kg.ha-1), provenientes de três formas de ureia (ureia convencional, ureia com inibidor de urease e ureia protegida). A adubação nitrogenada de cobertura foi realizada durante o estádio de perfilhamento, aos 20 dias após a emergência. Avaliaram-se: o teor de nitrogênio nas sementes, massa de 1000 sementes, germinação e vigor (primeira contagem de germinação, teste de frio, emergência de plântulas no campo, massa seca de plântulas, envelhecimento acelerado e condutividade elétrica). A linhagem IWT 04008 e a cultivar BRS Pardela apresentam sementes com qualidade fisiológica superior à cultivar BRS 208. As formas de ureia e as doses de nitrogênio em cobertura não alteram a qualidade fisiológica das sementes de diferentes genótipos de trigo.
\end{abstract}

Termos de indexação: Triticum aestivum L., ureia protegida, inibidor de urease, germinação, vigor de sementes.

\section{Forms of urea and nitrogen levels in topdressing on the physiological quality of wheat seeds}

\begin{abstract}
Besides increasing productivity, nitrogen fertilization may have positives effects on seed physiological quality. The objective of this study was to evaluate the effect of different forms and levels of urea in top dressing fertilization on the physiological quality of wheat seed genotypes. Seeds of three wheat genotypes (BRS 208, BRS Pardela and IWT 04008) were evaluated for four levels of nitrogen fertilization $\left(0,40,80\right.$ and $\left.120 \mathrm{~kg}^{-h^{-1}}\right)$ in three forms of urea (conventional urea, urea with urease inhibitor and protected urea). The nitrogen fertilization was applied during tillering, 20 days after emergence. The seed nitrogen content, 1000 seed mass, germination and vigor (germination first count, cold test, seedling emergence in the field, dry weight of seedlings, accelerated aging and electrical conductivity) were evaluated. The IWT 04008 line and the cultivar BRS Pardela had seeds with a higher physiological quality than those of the cultivar BRS 208. The forms of urea and levels of nitrogen in topdressing did not affect seed physiological quality of the different wheat genotypes.
\end{abstract}

Index terms: Triticum aestivum L., protected urea, urease inhibitor, germination, seed vigor.

${ }^{1}$ Submetido em 21/01/2011. Aceito para publicação em 12/07/2011.

${ }^{2}$ Departamento de Agronomia, Universidade Estadual de Londrina (UEL), Caixa Postal 6001, 86051-990 - Londrina, PR, Brasil.

${ }^{3}$ Embrapa Soja, EPAMIG, Caixa Postal 311, 38001-970 - Uberaba, MG, Brasil.
${ }^{4}$ Faculdade de Ciências Agronômicas, UNESP, 18.610-307 - Botucatu, SP, Brasil.

*Autor para correspondência <andre.mateus@hotmail.com> 


\section{Introdução}

A semente pode determinar o sucesso ou fracasso da produção agrícola, pois nela está toda a potencialidade produtiva da planta. A qualidade da semente refere-se ao somatório dos atributos genéticos, físicos, fisiológicos e sanitários, sendo assim, a boa qualidade da semente pode ser observada no cultivo resultante pela uniformidade da população de plantas, ausência de moléstias transmitidas pela semente, elevado vigor e maior produtividade (Carvalho e Nakagawa, 2000).

A qualidade fisiológica da semente desperta atenção especial da pesquisa, no sentido de elucidar os mais variados aspectos referentes às informações sobre a viabilidade e o vigor da semente (Marcos Filho, 2005). Porém, muitos fatores podem afetar a qualidade das sementes, dentre eles, a adubação, visto que plantas adequadamente adubadas apresentam condições de produzir maior quantidade de sementes, aliada a maior qualidade (Sá, 1994).

A disponibilidade de nutrientes interfere na formação e composição química das sementes, afetando o seu metabolismo e vigor (Carvalho e Nakagawa, 2000). Dentre os nutrientes, o nitrogênio $(\mathrm{N})$ destaca-se pela participação na constituição de proteínas e em funções metabólicas essenciais para a planta. Assim, os benefícios da adubação nitrogenada podem ir além do aumento da produtividade, possivelmente estando também associados à qualidade fisiológica das sementes. Efeitos positivos sobre a qualidade fisiológica das sementes foram relatados para outras gramíneas, como milho (Imolesi et al., 2001; Bono et al., 2008) e painço (Abrantes et al., 2010). Nesse contexto, o nitrogênio tem importância relevante e os seus efeitos variam com as condições ambientais e o estádio de desenvolvimento da planta em que o fertilizante é aplicado (Carvalho e Nakagawa, 2000).

A adubação nitrogenada de cobertura é uma das práticas mais importantes de manejo de gramíneas, pois ajuda no crescimento e no desenvolvimento das plantas, aumentando a produtividade (Sangoi et al., 2007). O nitrogênio é fertilizante que mais onera o custo de produção no cultivo de cereais, sendo de grande importância a utilização de cultivares e práticas adequadas de manejo que proporcionem maior eficiência de aplicação, maior aproveitamento no nitrogênio no solo e maior acúmulo de nitrogênio nos grãos (Schuch et al., 2000).

Entre as diversas fontes utilizadas para a adubação de cobertura nitrogenada, a ureia é a mais concentrada ( $45 \%$ de N) e, consequentemente, a de menor custo por unidade de nitrogênio (Yano et al., 2005). No entanto, possui como característica desfavorável as elevadas perdas por volatilização, as quais são aumentadas pela presença de palhada sobre o solo e pela falta de chuva para a sua incorporação (Cantarella et al., 2008). Para contornar esse problema, nos últimos anos foram lançados no mercado produtos contendo ureia com aditivos. Com isso, as perdas por volatilização são minimizadas, principalmente em condições edafoclimáticas desfavoráveis.

As formas alternativas de uréia, além de aumentar a eficiência, podem retardar a disponibilização de nitrogênio, pelo atraso na hidrólise pelo inibidor de urease ou pela disponibilização gradual promovida pelo impedimento físico do polímero, disponibilizando no momento mais adequado para a melhoria da qualidade da semente (Bono et al., 2008).

O objetivo do trabalho foi avaliar o efeito de doses de adubação nitrogenada em cobertura, provenientes de diferentes formas de ureia, na qualidade fisiológica de sementes de genótipos de trigo.

\section{Material e Métodos}

Sementes de trigo de três genótipos da Embrapa Soja (cultivares BRS 208 e BRS Pardela e a linhagem promissora IWT 04008) foram produzidas em experimentos independentes, no sistema de plantio direto após a cultura da soja durante a safra 2008. Os genótipos utilizados são todos de ciclo médio (cerca de 60 a 70 dias da emergência ao espigamento) e apresentam características contrastantes de altura de plantas, capacidade de perfilhamento, potencial produtivo e qualidade industrial do grão (Bassoi et al. 2007).

Os cultivos foram realizados nas áreas experimentais da Embrapa Soja, em Londrina-PR e da Embrapa Transferência de Tecnologia, Escritório de Negócios de Ponta Grossa, em Ponta Grossa-PR. O solo da área experimental de Londrina é classificado como Latossolo Vermelho distroférrico e a altitude atinge $600 \mathrm{~m}$. O clima da região, segundo a classificação de Köppen, é Cfa, ou seja, clima subtropical com temperatura média de $18{ }^{\circ} \mathrm{C}$ a $22{ }^{\circ} \mathrm{C}$ (Caviglione et al., 2000). Durante todo o ciclo da cultura a precipitação pluvial foi de $312 \mathrm{~mm}$, mais $15 \mathrm{~mm}$ de irrigação, totalizando $327 \mathrm{~mm}$. O solo da área experimental de Ponta Grossa é caracterizado como Cambissolo Háplico distrófico e a altitude é de $870 \mathrm{~m}$. O clima da região, segundo a classificação de Köppen, é Cfb, ou seja, clima temperado propriamente dito (Caviglione et al., 2000). A precipitação pluvial durante todo o ciclo da cultura foi de 486 mm, e não utilizou-se irrigação. A análise química do solo das áreas experimentais de Londrina e Ponta Grossa é apresentada na Tabela 1. 
Tabela 1. Atributos químicos do solo da área experimental nas camadas de 0-10 e 10-20 $\mathrm{cm}$ de profundidade (Prof).

\begin{tabular}{ccccccccccc}
\hline $\begin{array}{c}\text { Prof } \\
\mathrm{cm}\end{array}$ & $\begin{array}{c}\mathrm{pH} \\
\mathrm{CaCl}_{2}\end{array}$ & $\begin{array}{c}\mathrm{P} \\
\mathrm{mg} \cdot \mathrm{dm}^{-3}\end{array}$ & $\mathrm{Ca}^{+2}$ & $\mathrm{Mg}^{+2}$ & $\begin{array}{c}\mathrm{K}^{+} \\
\mathrm{cmol}_{\mathrm{c}} \cdot \mathrm{dm}^{-3}\end{array}$ & $\mathrm{H}+\mathrm{Al}$ & $\mathrm{CTC}$ & $\begin{array}{c}\mathrm{V} \\
\%\end{array}$ & $\begin{array}{c}\mathrm{C} \\
\mathrm{g} \cdot \mathrm{dm}^{-3}\end{array}$ \\
\hline \multicolumn{8}{c}{} & \multicolumn{7}{c}{ Londrina } \\
\hline $00-10$ & 4,60 & 5,95 & 4,26 & 2,86 & 0,82 & 0,09 & 3,92 & 11,87 & 66,94 & 7,97 \\
$10-20$ & 4,51 & 6,53 & 3,88 & 2,66 & 0,59 & 0,09 & 4,11 & 11,24 & 63,46 & 7,54 \\
\hline \multicolumn{7}{c}{ Ponta Grossa } \\
\hline $00-10$ & 5,57 & 12,57 & 5,78 & 2,04 & 0,19 & 0,02 & 4,39 & 12,39 & 64,50 & 12,86 \\
$10-20$ & 5,03 & 6,09 & 5,88 & 2,08 & 0,16 & 0,04 & 5,93 & 14,05 & 57,75 & 13,50 \\
\hline
\end{tabular}

A semeadura foi realizada dentro do período indicado para cada local (Fronza et al., 2008) em Londrina no dia 24/04/2008 e em Ponta Grossa no dia 09/07/2008, visando à obtenção de uma densidade de aproximadamente 300 plantas. $\mathrm{m}^{-2}$. A adubação de nitrogênio, fósforo e potássio (NPK) na semeadura foi de $20 \mathrm{~kg} \cdot \mathrm{ha}^{-1} \mathrm{de} \mathrm{N}, 50 \mathrm{~kg} \cdot \mathrm{ha}^{-1} \mathrm{de}_{2} \mathrm{O}_{5}$ e $50 \mathrm{~kg} \cdot \mathrm{ha}^{-1}$ de $\mathrm{K}_{2} \mathrm{O}$. A adubação nitrogenada de cobertura foi realizada no estádio de perfilhamento, aos 20 dias após a emergência. Os tratamentos consistiram em quatro doses de $\mathrm{N}$ em cobertura $\left(0,40,80\right.$ e $\left.120 \mathrm{~kg} \cdot \mathrm{ha}^{-1}\right)$ e três formas de ureia (ureia convencional $45 \%$ de $\mathrm{N}$, ureia com inibidor de urease-SuperN ${ }^{\circledR} 45 \%$ de $\mathrm{N}$ e ureia protegida-Kim Coat ${ }^{\circledR}$ $41 \%$ de N). Foi utilizado o delineamento experimental de blocos casualizados, em esquema fatorial $3 \times 4$, com quatro repetições. Cada genótipo, em cada local, constituiu um experimento, totalizando seis experimentos. A parcela experimental foi constituída por dez linhas de seis metros de comprimento, com espaçamento entre linhas de 0,20 m. A área útil de cada parcela experimental foi composta pelas seis linhas centrais, desprezando-se $0,75 \mathrm{~m}$ nas extremidades, totalizando $5,4 \mathrm{~m}^{2}$.

Os tratos culturais foram realizados de acordo com as indicações técnicas para a cultura do trigo no Estado do Paraná (Fronza et al., 2008), sendo a área monitorada semanalmente e as aplicações de fungicidas realizadas no aparecimento dos primeiros sintomas, visando proporcionar as melhores condições possíveis para o desenvolvimento da cultura.

As sementes dos três genótipos foram colhidas no mesmo dia em Londrina (02/09/2008) e em Ponta Grossa (25/11/2008), ambos na maturação de colheita com grau de umidade em torno de $15 \%$. Após a colheita foi realizada a pré-limpeza das sementes em peneira de crivo oblongo de $1,75 \times 20,0 \mathrm{~mm}$ que, posteriormente, foram armazenadas em câmara fria, a temperatura em torno de $12^{\circ} \mathrm{C}$, até a realização das análises, as quais foram realizadas no Laboratório de Fitotecnia na Universidade Estadual de Londrina, Londrina-PR, no período de março a maio de 2009.
A qualidade fisiológica das sementes foi avaliada em cada parcela obtida no campo.

A massa de 1000 sementes foi determinada por meio da massa de duas amostras de 100 sementes para cada parcela experimental utilizando balança com precisão de duas casas decimais.

Para o teste de germinação utilizaram-se duas repetições de 50 sementes de cada parcela, que foram semeadas em duas folhas e cobertas com uma folha de papel tipo "germitest", umedecido com volume de água equivalente a 2,5 vezes a massa do papel e mantido em câmara de germinação a $20^{\circ} \mathrm{C}$ com 16 horas de luz diária. As avaliações foram realizadas com base nas Regras para Análise de Sementes (Brasil, 2009) aos quatro (1 $1^{\text {a }}$ contagem) e sete ( $2^{\mathrm{a}}$ contagem) dias após a instalação do teste, contabilizando-se porcentagem de plântulas normais.

Para o teste de frio foi utilizada a metodologia descrita por Krzyzanowski et al. (1999) adaptada para a cultura do trigo. Foram utilizadas duas repetições de 50 sementes de cada parcela, semeadas em duas folhas e cobertas com uma folha em papel "germitest", umedecido com volume de água destilada equivalente a 2,5 vezes a massa do papel e mantidas em câmara de refrigeração à temperatura de $5{ }^{\circ} \mathrm{C}$, por sete dias, sendo depois transferidas para germinador à temperatura de $20^{\circ} \mathrm{C}$. Após quatro dias foi avaliada a porcentagem de plântulas normais.

Para o teste de emergência de plântulas no campo utilizaram-se 50 sementes de cada parcela, com espaçamento de $3 \mathrm{~cm}$ entre semente e $4 \mathrm{~cm}$ entre linha. A contagem foi realizada 14 dias após a semeadura, considerando todas as plântulas emergidas, independentemente do estádio de desenvolvimento. As plântulas emergidas foram coletadas, lavadas, colocadas em sacos de papel e levadas à estufa de circulação de ar forçada, à temperatura de $65^{\circ} \mathrm{C}$, até atingirem massa constante para determinação da massa seca (MSP) de plântula, expressando a média individual de MSP em mg.

No teste de envelhecimento acelerado utilizaram-se 
65 sementes de cada parcela distribuídas em camada única sobre bandeja de tela metálica fixada no interior de caixa plástica, tipo gerbox, contendo $40 \mathrm{~mL}$ de água destilada. As caixas foram tampadas e levadas a câmara de envelhecimento acelerado a $41^{\circ} \mathrm{C}$, por 72 horas, conforme metodologia proposta pela Association of Official Seed Analysts (1983). Após esse período, 15 sementes foram utilizadas para determinação do teor de água e 50 sementes foram submetidas ao teste de germinação, sendo realizada uma única contagem cinco dias após a instalação, contabilizando-se a porcentagem de plântulas normais.

Para o teste de condutividade elétrica foram utilizadas 50 sementes de cada parcela, previamente pesadas e colocadas para embeber em $75 \mathrm{~mL}$ de água destilada por um período de 24 horas, a $25{ }^{\circ} \mathrm{C}$ (Krzyzanowski et al., 1999). Em seguida, procedeu-se a leitura da condutividade elétrica da solução de embebição em condutivímetro modelo Digimed D31, calibrado, expressando os resultados em $\mu \mathrm{S} . \mathrm{cm}^{-1} \cdot \mathrm{g}^{-1}$.
O teor de nitrogênio nos grãos foi determinado seguindo a metodologia de Kjeldahl, utilizando uma amostra de 0,2 gramas de grãos moídos e com granulometria inferior a 0,2 mm expressando os resultados em g.kg-1 (Malavolta, 2006).

A análise exploratória dos dados foi realizada para verificar o atendimento das pressuposições da análise de variância e a presença de "outliers". Além disso, foi realizada a relação entre o maior e menor resíduo antes de se proceder à análise conjunta dos experimentos para cada local. Os dados foram submetidos à análise de variância e as médias de formas de ureia foram comparadas pelo teste de Tukey a $5 \%$, e os dados de doses foram submetidos à análise de regressão até segundo grau.

\section{Resultados e Discussão}

As interações entre dose e forma, genótipo e dose, genótipo, forma e genótipo e dose e forma de ureia não foram significativas para as características avaliadas (Tabela 2).

Tabela 2. Resumo da análise de variância para as características avaliadas em genótipos de trigo, em função de formas de ureia e doses de nitrogênio aplicadas em cobertura.

\begin{tabular}{|c|c|c|c|c|c|c|c|c|c|}
\hline \multicolumn{10}{|c|}{ Londrina } \\
\hline F V & TPG & $\mathrm{PC}$ & $\mathrm{TF}$ & $\mathrm{EC}$ & MSP & EA & $\mathrm{CE}$ & MMS & TNS \\
\hline $\mathrm{BL}(\mathrm{GEN})$ & $0,815^{\mathrm{ns}}$ & $0,485^{\mathrm{ns}}$ & $0,419^{\mathrm{ns}}$ & $0,000^{* *}$ & $0,487^{\mathrm{ns}}$ & $0,050^{\mathrm{ns}}$ & $0,063^{\mathrm{ns}}$ & $0,429^{\mathrm{ns}}$ & $0,342^{\mathrm{ns}}$ \\
\hline GEN & $0,519^{\mathrm{ns}}$ & $0,108^{\mathrm{ns}}$ & $0,000^{* *}$ & $0,000^{* *}$ & $0,000 * *$ & $0,013 *$ & $0,000^{* *}$ & $0,000 * *$ & $0,442^{\mathrm{ns}}$ \\
\hline DOSE & $0,936^{\mathrm{ns}}$ & $0,970^{\mathrm{ns}}$ & $0,148^{\mathrm{ns}}$ & $0,624^{\mathrm{ns}}$ & $0,928^{\mathrm{ns}}$ & $0,774^{\mathrm{ns}}$ & $0,000^{* *}$ & $0,000 * *$ & $0,0492 *$ \\
\hline FORMA & $0,187^{\mathrm{ns}}$ & $0,112^{\mathrm{ns}}$ & $0,752^{\mathrm{ns}}$ & $0,403^{\mathrm{ns}}$ & $0,659^{\mathrm{ns}}$ & $0,013 *$ & $0,586^{\mathrm{ns}}$ & $0,659^{\mathrm{ns}}$ & $0,487^{\mathrm{ns}}$ \\
\hline $\mathrm{DO} * \mathrm{FO}$ & $0,956^{\mathrm{ns}}$ & $0,713^{\mathrm{ns}}$ & $0,416^{\mathrm{ns}}$ & $0,537^{\mathrm{ns}}$ & $0,304^{\mathrm{ns}}$ & $0,768^{\mathrm{ns}}$ & $0,450^{\mathrm{ns}}$ & $0,647^{\mathrm{ns}}$ & $0,127^{\mathrm{ns}}$ \\
\hline GEN*DO & $0,693^{\mathrm{ns}}$ & $0,653^{\mathrm{ns}}$ & $0,352^{\mathrm{ns}}$ & $0,847^{\mathrm{ns}}$ & $0,324^{\mathrm{ns}}$ & $0,652^{\mathrm{ns}}$ & $0,120^{\mathrm{ns}}$ & $0,899^{\mathrm{ns}}$ & $0,050^{\mathrm{ns}}$ \\
\hline GEN*FO & $0,210^{\mathrm{ns}}$ & $0,124^{\mathrm{ns}}$ & $0,272^{\mathrm{ns}}$ & $0,300^{\mathrm{ns}}$ & $0,981^{\mathrm{ns}}$ & $0,770^{\mathrm{ns}}$ & $0,078^{\mathrm{ns}}$ & $0,215^{\mathrm{ns}}$ & $0,925^{\text {ns }}$ \\
\hline GEN*DO*FO & $0,912^{\mathrm{ns}}$ & $0,890^{\mathrm{ns}}$ & $0,700^{\mathrm{ns}}$ & $0,851^{\mathrm{ns}}$ & $0,165^{\mathrm{ns}}$ & $0,095^{\mathrm{ns}}$ & $0,051^{\mathrm{ns}}$ & $0,666^{\mathrm{ns}}$ & $0,344^{\mathrm{ns}}$ \\
\hline CV (\%) & 6,3 & 10,2 & 8,1 & 20,9 & 16,3 & 36,3 & 7,3 & 4,3 & 10,9 \\
\hline \multicolumn{10}{|c|}{ Ponta Grossa } \\
\hline F V & TPG & $\mathrm{PC}$ & $\mathrm{TF}$ & $\mathrm{EC}$ & MSP & EA & $\mathrm{CE}$ & MMS & TNS \\
\hline BL(GEN) & $0,396^{\mathrm{ns}}$ & $0,183^{\mathrm{ns}}$ & $0,821^{\mathrm{ns}}$ & $0,195^{\mathrm{ns}}$ & $0,396^{\mathrm{ns}}$ & $0,125^{\mathrm{ns}}$ & $0,084^{\mathrm{ns}}$ & $0,142^{\mathrm{ns}}$ & $0,979^{\mathrm{ns}}$ \\
\hline GEN & $0,000 * *$ & $0,000^{* *}$ & $0,000 * *$ & $0,000^{* *}$ & $0,000 * *$ & $0,000^{* *}$ & $0,000^{* *}$ & $0,000^{* *}$ & $0,000^{* *}$ \\
\hline DOSE & $0,851^{\mathrm{ns}}$ & $0,688^{\mathrm{ns}}$ & $0,520^{\mathrm{ns}}$ & $0,636^{\mathrm{ns}}$ & $0,257^{\mathrm{ns}}$ & $0,788^{\mathrm{ns}}$ & $0,852^{\mathrm{ns}}$ & $0,296^{\mathrm{ns}}$ & $0,066^{\mathrm{ns}}$ \\
\hline FORM & $0,492^{\mathrm{ns}}$ & $0,485^{\mathrm{ns}}$ & $0,406^{\mathrm{ns}}$ & $0,835^{\mathrm{ns}}$ & $0,163^{\mathrm{ns}}$ & $0,444^{\mathrm{ns}}$ & $0,535^{\mathrm{ns}}$ & $0,875^{\text {ns }}$ & $0,948^{\text {ns }}$ \\
\hline $\mathrm{DO} * \mathrm{FO}$ & $0,355^{\mathrm{ns}}$ & $0,059^{\mathrm{ns}}$ & $0,592^{\mathrm{ns}}$ & $0,367^{\mathrm{ns}}$ & $0,141^{\mathrm{ns}}$ & $0,346^{\mathrm{ns}}$ & $0,842^{\mathrm{ns}}$ & $0,379^{\mathrm{ns}}$ & $0,540^{\mathrm{ns}}$ \\
\hline GEN*DO & $0,840^{\mathrm{ns}}$ & $0,932^{\mathrm{ns}}$ & $0,825^{\mathrm{ns}}$ & $0,586^{\mathrm{ns}}$ & $0,589^{\mathrm{ns}}$ & $0,654^{\mathrm{ns}}$ & $0,773^{\mathrm{ns}}$ & $0,610^{\mathrm{ns}}$ & $0,504^{\mathrm{ns}}$ \\
\hline GEN*FO & $0,695^{\mathrm{ns}}$ & $0,440^{\mathrm{ns}}$ & $0,188^{\mathrm{ns}}$ & $0,649^{\mathrm{ns}}$ & $0,481^{\mathrm{ns}}$ & $0,714^{\mathrm{ns}}$ & $0,407^{\mathrm{ns}}$ & $0,482^{\mathrm{ns}}$ & $0,640^{\mathrm{ns}}$ \\
\hline GEN*DO*FO & $0,675^{\mathrm{ns}}$ & $0,565^{\mathrm{ns}}$ & $0,817^{\mathrm{ns}}$ & $0,713^{\mathrm{ns}}$ & $0,340^{\mathrm{ns}}$ & $0,379^{\mathrm{ns}}$ & $0,991^{\mathrm{ns}}$ & $0,838^{\mathrm{ns}}$ & $0,049^{\mathrm{ns}}$ \\
\hline CV (\%) & 7,6 & 9,9 & 12,1 & 11,1 & 22,0 & 22,5 & 14,0 & 2,3 & 8,4 \\
\hline
\end{tabular}

ns, ** e *: não significativo e significativo a $1 \%$ e $5 \%$ de probabilidade, respectivamente, pelo teste $\mathrm{F}$.

BL: bloco, GEN: genótipo, DO: dose de N, FO: forma de ureia, CV: coeficiente de variação, TPG: Teste de germinação, PC: primeira contagem, TF: teste de frio, EC: Emergência em campo, MSP: massa seca de plântula, EA: envelhecimento acelerado, CE: condutividade elétrica, MMS: massa de mil sementes, TNS: teor de nitrogênio na semente. 
Para os experimentos conduzidos em Londrina-PR, o efeito de genótipos foi significativo para as características porcentagem no teste de frio, emergência em campo, massa seca de plântulas do teste de emergência em campo, condutividade elétrica, massa de mil sementes e teste de envelhecimento acelerado (Tabela 2); não foi constatado efeito de genótipos para o teste de germinação, primeira contagem de germinação e teor de nitrogênio na semente. $\mathrm{O}$ efeito de genótipos em Ponta Grossa foi altamente significativo, para todas as características avaliadas. Em geral, para os dois locais, as sementes da cultivar BRS Pardela e a linhagem IWT 04008 apresentaram qualidade fisiológica superior às da cultivar BRS 208 (Tabela 3). Como as sementes foram colhidas no mesmo dia e com mesmo teor de água, essa diferença talvez possa ser atribuída a efeitos genéticos não avaliados nesse experimento, uma vez que o resultado não se coorrelaciona com o teor de nitrogênio na semente e este não diferiu entre os genótipos em Londrina e foi superior nas cultivares BRS 208 e BRS Pardela em Ponta Grossa.

Tabela 3. Valores médios dos atributos de qualidade fisiológica de sementes em genótipos de trigo.

\begin{tabular}{|c|c|c|c|c|c|c|c|c|c|}
\hline & TPG & $\mathrm{PC}$ & $\mathrm{TF}$ & $\mathrm{EC}$ & MSP & EA & CE & MMS & TNS \\
\hline Genótipos & $(\%)$ & $(\%)$ & $(\%)$ & $(\%)$ & $(\mathrm{mg})$ & $(\%)$ & $\left(\mu \mathrm{S} . \mathrm{cm}^{-1} \cdot \mathrm{g}^{-1}\right)$ & (g) & $\left(\mathrm{g} \cdot \mathrm{kg}^{-1}\right)$ \\
\hline \multicolumn{10}{|c|}{ Londrina } \\
\hline BRS 208 & $90 \mathrm{a}$ & $40 \mathrm{a}$ & $69 \mathrm{~b}$ & $65 \mathrm{~b}$ & $13,1 \mathrm{a}$ & $43 \mathrm{ab}$ & $54,5 \mathrm{~b}$ & $32,6 \mathrm{a}$ & $22,5 \mathrm{a}$ \\
\hline Pardela & $91 \mathrm{a}$ & $40 \mathrm{a}$ & $85 \mathrm{a}$ & $48 \mathrm{c}$ & $9,5 \mathrm{~b}$ & $49 \mathrm{a}$ & $51,6 \mathrm{a}$ & $31,3 \mathrm{~b}$ & $23,1 \mathrm{a}$ \\
\hline IWT 04008 & $90 \mathrm{a}$ & $41 \mathrm{a}$ & $84 \mathrm{a}$ & $73 \mathrm{a}$ & $13,0 \mathrm{a}$ & $39 \mathrm{~b}$ & $50,1 \mathrm{a}$ & $29,6 \mathrm{c}$ & $22,9 \mathrm{a}$ \\
\hline \multicolumn{10}{|c|}{ Ponta Grossa } \\
\hline BRS 208 & $79 \mathrm{~b}$ & $34 \mathrm{c}$ & $73 \mathrm{~b}$ & $71 \mathrm{~b}$ & $17,6 \mathrm{a}$ & $51 \mathrm{~b}$ & $33,9 \mathrm{a}$ & $39,5 \mathrm{~b}$ & $26,1 \mathrm{a}$ \\
\hline Pardela & $85 \mathrm{a}$ & $38 \mathrm{~b}$ & $83 \mathrm{a}$ & $81 \mathrm{a}$ & $13,9 \mathrm{c}$ & $66 \mathrm{a}$ & $29,2 \mathrm{~b}$ & $40,2 \mathrm{a}$ & $26,8 \mathrm{a}$ \\
\hline IWT 04008 & $87 \mathrm{a}$ & $40 \mathrm{a}$ & $85 \mathrm{a}$ & $84 \mathrm{a}$ & $15,7 \mathrm{~b}$ & $60 \mathrm{a}$ & $33,6 \mathrm{a}$ & $38,1 \mathrm{c}$ & $23,5 \mathrm{~b}$ \\
\hline
\end{tabular}

As médias dentro de cada coluna seguidas da mesma letra não diferem entre si, pelo teste de Tukey, a 5\% de probabilidade. TPG: Teste de germinação, PC: primeira contagem, TF: teste de frio, EC: Emergência em campo, MSP: massa seca de plântula, EA: envelhecimento acelerado, CE: condutividade elétrica, MMS: massa de mil sementes, TNS: teor de nitrogênio na semente.

Não foram observados efeitos significativos em função de doses de $\mathrm{N}$ em cobertura em Ponta Grossa (Tabela 2). Para os experimentos conduzidos em Londrina o efeito de doses foi signicativo apenas para condutividade elétrica, massa de mil sementes e teor de nitrogênio na semente. A massa de mil sementes decresceu com o aumento das doses de nitrogênio, conforme uma curva de tendência quadrática (Figura 1), esse resultado pode ser consequência do acamamento ocorrido na fase de enchimento de grãos, o qual prejudicou o enchimento, e também, devido ao provável aumento no número de sementes e perfilhos por área, com o incremento das doses de N. A condutividade elétrica teve um comportamento crescente, de tendência quadrática, em função do incremento das doses de nitrogênio aplicadas em cobertura (Figura 1). Isso pode ter sido causado pelo maior acúmulo de nutrientes, não sendo possível concluir que este efeito está associado a uma maior desintegração de membranas da semente. $\mathrm{O}$ teor de nitrogênio na semente aumentou linearmente com o incremento de doses de $\mathrm{N}$ em cobertura, esse resultado possivelmente está relacionado com a redução na massa da semente, concentrando mais proteína na semente, pois para Ponta Grossa não foram observados efeitos em função de doses de $\mathrm{N}$ em cobertura (Tabela 2).

De modo geral, o incremento nas doses de nitrogênio não proporcionou melhoria na qualidade fisiológica nem no teor de nitrogênio na semente. Esse resultado deve-se, em parte, às condições do solo dos dois locais, pois ambos os solos possuem elevada fertilidade associada ao manejo sob sistema de plantio direto e, também, pela rotação de cultura com uma leguminosa, que possivelmente tenha deixado no solo e na palhada nutrientes, especialmente o nitrogênio, para a cultura do trigo. Com isso, mesmo na testemunha com aplicação de apenas $20 \mathrm{~kg} \cdot \mathrm{ha}^{-1}$ de $\mathrm{N}$ na semeadura e sem a adubação de cobertura, foi possível produzir sementes com boa qualidade fisiológica. A não influência da adubação nitrogenada na qualidade fisiológica de sementes devese ao fato de que as plantas tem a tendência de numa condição de menor disponibilidade de nutrientes priorizar a preservação da espécie, isso significa, produzir menos, 
mas sementes com qualidade, por isso não é evidenciado o efeito da adubação (Delouche, 1980). Esses resultados corroboram com obtidos em outras culturas, tais como aveia- preta (Silva et al., 2001), aveia branca (Kolchinski e Schuch, 2004) e arroz (Souza et al., 2010). Contudo contraria os resultados obtidos por Imolesi et al. (2001) e Bono et al. (2008), em sementes de milho, e Abrantes et al. (2010), em sementes de painço, que observaram aumento na qualidade fisiológica com a aplicação de nitrogênio.
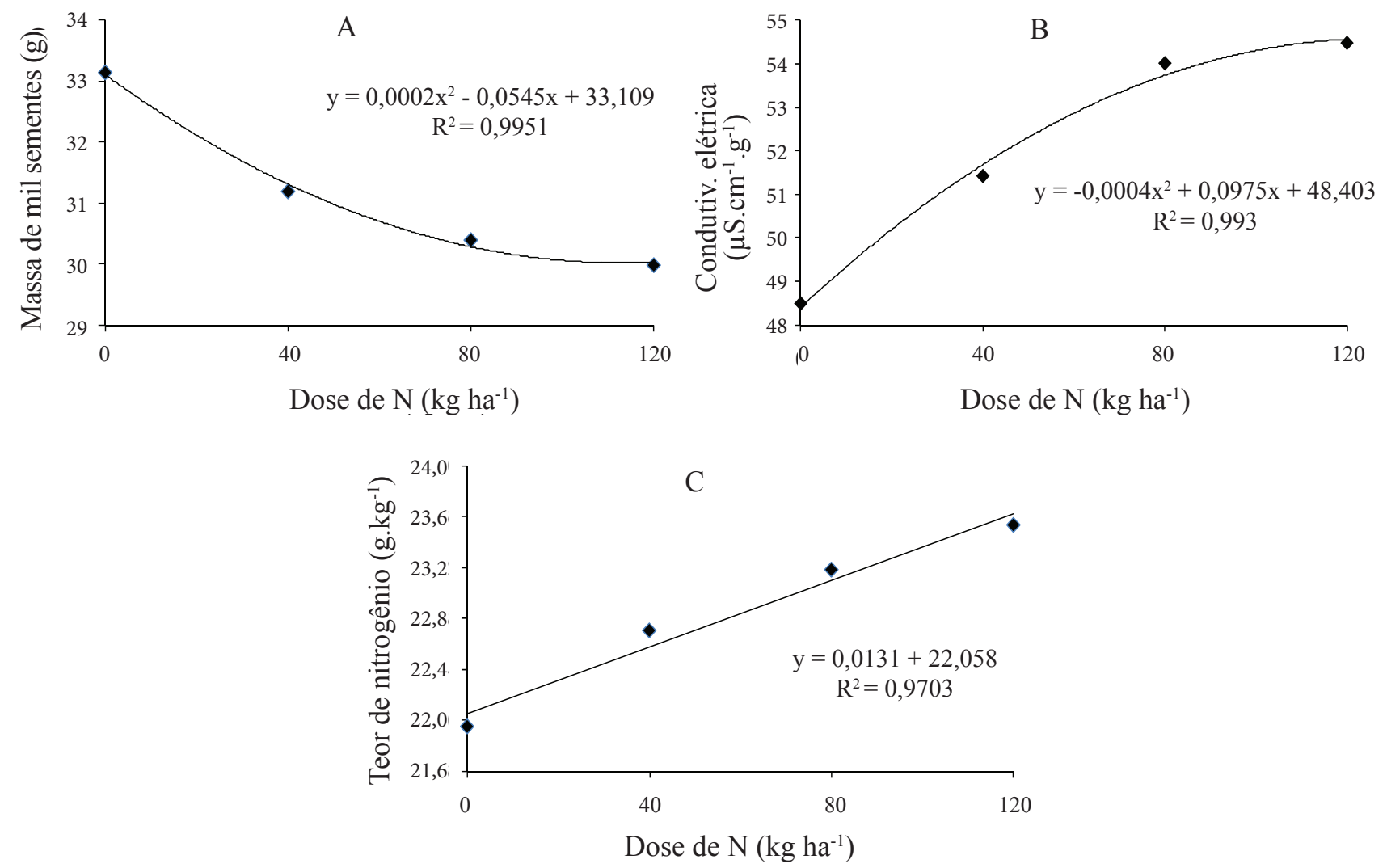

Figura 1. Massa de mil sementes (a), condutividade elétrica (b) e teor de nitrogênio na semente (c) em genótipos de trigo, em função de doses de nitrogênio aplicadas em cobertura.

Não foi observado efeito significativo das formas de ureia para a maioria das características analisadas, com exceção no teste de envelhecimento acelerado das sementes oriundas dos experimentos conduzidos em Londrina (Tabela 2). Para essa característica, a ureia recoberta com polímero resultou em maior vigor de sementes que a ureia convencional, mas ambas não diferiram da ureia recoberta com inibidor de urease (Tabela 4). Esperava-se que a utilização de formas alternativas de ureia promovesse aumento da eficiência do aproveitamento da ureia, com isso, uma maior quantidade de nitrogênio estaria disponível para o trigo. Além disso, as formas alternativas de ureia podem retardar a disponibilização de nitrogênio da ureia, pelo atraso na hidrólise pelo inibidor de urease ou pela disponibilização gradual promovida pelo polímero, disponibilizando no momento mais adequado para a melhoria da qualidade da semente (Bono et al., 2008). Isso poderia ser detectado pelo aumento do teor de nitrogênio e pela qualidade fisiológica da semente. No entanto isso não foi observado, provavelmente devido às condições climáticas ocorridas após a adubação nitrogenada de cobertura nos experimentos. Em Londrina, houve uma irrigação de $15 \mathrm{~mm}$ após seis horas e, em Ponta Grossa, a precipitação pluvial ocorreu após 17 dias da adubação nitrogenada. Além disso, o aumento na eficiência da utilização da ureia com o uso das formas diferenciadas não foi suficiente para influenciar as características avaliadas, uma vez que nem mesmo as altas doses utilizadas proporcionaram diferença significativa. 
Tabela 4. Valores médios dos atributos de qualidade fisiológica de sementes em genótipos de trigo, em função das formas de ureia aplicadas em cobertura.

\begin{tabular}{cccccccccc}
\hline Forma & $\begin{array}{c}\text { TPG } \\
(\%)\end{array}$ & $\begin{array}{c}\text { PC } \\
(\%)\end{array}$ & $\begin{array}{c}\text { TF } \\
(\%)\end{array}$ & $\begin{array}{c}\text { EC } \\
(\%)\end{array}$ & $\begin{array}{c}\text { MSP } \\
(\mathrm{mg})\end{array}$ & $\begin{array}{c}\text { EA } \\
(\%)\end{array}$ & $\begin{array}{c}\text { CE } \\
\left(\mu \mathrm{S} \cdot \mathrm{cm}^{-1} \cdot \mathrm{g}^{-1}\right)\end{array}$ & $\begin{array}{c}\text { MMS } \\
(\mathrm{g})\end{array}$ & $\begin{array}{c}\text { TNS } \\
\left(\mathrm{g} \cdot \mathrm{kg}^{-1}\right)\end{array}$ \\
\hline Ureia & 90 & 40 & 79 & 60 & 11,9 & $39 \mathrm{~b}$ & 52,5 & 31,1 & 22,5 \\
Ureia+inibidor & 90 & 40 & 80 & 63 & 11,6 & $45 \mathrm{ab}$ & 52,1 & 31,1 & 22,8 \\
Ureia+polímero & 92 & 41 & 79 & 63 & 11,9 & $48 \mathrm{a}$ & 51,7 & 31,3 & 23,2 \\
\hline & & \multicolumn{7}{c}{ Ponta Grossa } \\
\hline Ureia & 84 & 38 & 82 & 79 & 15,8 & 60 & 31,8 & 39,3 & 25,4 \\
Ureia+inibidor & 84 & 38 & 81 & 79 & 16,4 & 57 & 32,1 & 39,2 & 25,4 \\
Ureia+polímero & 83 & 37 & 79 & 78 & 15,0 & 60 & 32,8 & 39,3 & 25,5 \\
\hline
\end{tabular}

As médias dentro de cada coluna seguidas da mesma letra não diferem entre si, pelo teste de Tukey, a $5 \%$ de probabilidade. TPG: teste de germinação, PC: primeira contagem, TF: teste de frio, EC: Emergência em campo, MSP: massa seca de plântula, EA: envelhecimento acelerado, CE: condutividade elétrica, MMS: massa de mil sementes, TNS: teor de nitrogênio na semente.

\section{Conclusões}

A linhagem IWT 04008 e a cultivar BRS Pardela resultam em sementes com qualidade fisiológica superior à cultivar BRS 208 .

As formas de ureia e as doses de nitrogênio aplicadas em cobertura não alteram a qualidade fisiológica das sementes de diferentes genótipos de trigo.

\section{Referências}

ABRANTES, F.L.; KULCZYNSKI, S.M.; SORATTO, R.P.; BARBOSA, M.M.M. Nitrogênio em cobertura e qualidade fisiológica e sanitária de sementes de painço (Panicum miliaceum L.). Revista Brasileira de Sementes, v.32, n.3, p.106-115, 2010. http://www.scielo.br/pdf/rbs/ v32n3/v32n3a12.pdf

ASSOCIATION OF OFFICIAL SEED ANALYSTS. Seed vigour testing handbook. East Lansing: AOSA, 1983. 93p. (Contribuition, 32).

BASSOI, M.C.; BRUNETTA, D.; TAVARES, L.C.V; MIRANDA, L.C.; BECKERT, O.P.; SCHEEREN, P.L.; MIRANDA, M.Z.; RIEDE, C.R.;CAMPOS, L.A.C.; SHIOGA, P.S.; SCHOLZ, M.B.S.; OKUYAMA, L.A.; POLA, J.N.; AZAMBUJA, J.R.S. Cultivares de trigo Embrapa e Iapar. Londrina: Embrapa Soja, 2007. 68p. (Embrapa Soja. Documentos, 282).

BONO, J.A.M.; RODRIGUES A.P.D.C.; MAUAD, M.; ALBUQUERQUE, J.C.; YAMAMOTO C.R.; CHERMOUTH, K.S.; FREITA, M.E. Modo de aplicação de fertilizantes nitrogenados na qualidade fisiológica de sementes de milho. Agrarian, v.1, n.2, p.91-102, 2008. http://www.scielo.br/pdf/rbs/v32n3/v32n3a12.pdf

BRASIL, Ministério da Agricultura, Pecuária e Abastecimento. Regras para análise de sementes. Ministério da Agricultura, Pecuária e Abastecimento. Secretaria de Defesa Agropecuária. Brasília, DF: MAPA/ACS, 2009. 395p. http://www.agricultura.gov.br/arq_editor/file/laborat\%c3\%b3rio/ sementes $/$ regras $\% 20$ para $\% 20$ analise $\% 20 \mathrm{de} \% 20$ sementes.pdf

CANTARELlA, H.; TRIVELIN, P.C.O.; CONTIN, T.L.M.; DIAS, F.L.F.; ROSSETTO, R.; MARCELINO, R.; COIMBRA, R.B.; QUAGGIO, J.A. Ammonia volatilisation from urease inhibitor-treated urea applied to sugarcane trash blankets. Scientia Agricola, v.65, n.4, p.397-401, 2008. http://www.scielo.br/pdf/sa/v65n4/11.pdf

CARVALHO, N.M.; NAKAGAWA, J. Sementes: ciência, tecnologia e produção. Jaboticabal: FUNEP, 2000. 429p.

CAVIGLIONE, J.H.; KIIHL, L.R.B.; CARAMORI, P.H.; OLIVEIRA, D. Cartas climáticas do Paraná. Londrina : IAPAR, 2000. CD. http:// www.iapar.pr.gov.br/modules/conteudo/conteudo.php?conteudo=677

DELOUCHE, J.C. Environmental effects on seed development and seed quality. Hort Science, v.15, n.6, p.775-780, 1980.

FRONZA, V.; CAMPOS, L.A.C.; RIEDE, C.R. (Org.) Informações técnicas para a safra 2008: trigo e triticale. Londrina: Embrapa Soja, 2008. (Documentos, 301) 147p.

IMOLESI, A.S.; VON PINHO, E.V.R.; PINHO, R.G.V.; VIEIRA, M.G.G.C.; CORRÊA, R.S.B. Influência da adubação nitrogenada na qualidade fisiológica das sementes de milho. Ciência e Agrotecnologia, v.25, n.5, p.1119-1126, 2001. http://www.editora.ufla.br/site/_adm/ upload/revista/25-5-2001_10.pdf

KOLCHINSKI, E.M.; SCHUCH, L.O.B. Relação entre a adubação nitrogenada e a qualidade de grãos e de sementes em aveia branca. Ciência Rural, v.34, n.2, p.379-383, 2004.

KRZYZANOWSKI, F.C.; VIEIRA, R.D.; FRANÇA-NETO, J.B. (Ed.). Vigor de sementes: conceitos e testes. Londrina: ABRATES, 1999. 218p.

MALAVOLTA, E. Manual de nutrição de plantas. São Paulo: Agronômica Ceres, 2006. 638p. 
MARCOS-FILHO, J. Fisiologia de sementes de plantas cultivadas. Piracicaba: FEALQ, v.12, 2005. 495p.

SÁ, M.E. Importância da adubação nitrogenada na qualidade das sementes. In: SÁ, M.E.; BUZZETI, S. Importância da adubação na qualidade dos produtos agrícolas. São Paulo: Ícone, 1994. Cap. 6, p.65-98.

SANGOI, L.; BERNS, A.C.; ALMEIDA, M.L.; ZANIM, C.G.; SCHWEITZER, C. Características agronômicas de cultivares de trigo em resposta à época da adubação nitrogenada de cobertura. Ciência Rural, v.37, n.6, p.1564-1570, 2007. http://www.scielo.br/pdf/cr/v37n6/ a10v37n6.pdf

SCHUCH, L.O.B; NEDEL, J.L.; ASSIS, F.N.; MAIA, M.S. Vigor de sementes de populações de aveia preta: II. Desempenho e utilização de nitrogênio. Scientia Agricola, v.57, n.1, p.121-127, 2000. http://www. scielo.br/scielo.php?pid=S0103-90162000000100020\&script=sci_arttext
SILVA, R.H.; ZUCARELI, C.; NAKAGAWA, J.; SILVA, R.A.; CAVARIANI, C. Doses e épocas de aplicação do nitrogênio na produção e qualidade de sementes de aveia-preta. Revista Brasileira de Sementes, v.23, n.2, p.51-55, 2001. http://www.abrates.org.br/revista/artigos/2001/ v23n2/artigo07.pdf

SOUZA, L.C.D.; SÁ, M.E.; MARTINS, H.S.D.; ABRANTES, F.L.; SILVA, M.P.; ARRUDA, N. Produtividade e qualidade de sementes de arroz em resposta a doses de calcário e nitrogênio. Revista Trópica, v.4, n.2, p.27-35, 2010. http://www.periodicoseletronicos.ufma.br/index.php/ ccaatropica/article/view/153/101

YANO, G.T.; TAKAHASHI, H.T.; WATANABE, T.S. Avaliação de fontes de nitrogênio e épocas de aplicação em cobertura para o cultivo do trigo. Semina: Ciências Agrárias, v.26, n.2, p.141-148, 2005. http:// www.scielo.br/pdf/cr/v37n6/a10v37n6.pdf 